\title{
Artigo \\ Protótipo de sistema direcionador de vento automatizado para aerogeradores de eixo horizontal
}

\author{
Bruno M. M. Silva ${ }^{[1]}$, Lucas V. F. Rodrigues ${ }^{[2]}$, Stephano M. N. da Silva ${ }^{[3]}$, Fabiana Karla de Oliveira \\ Martins Varella Guerra ${ }^{[4]}$, Marcelo Roberto Bastos Guerra Vale ${ }^{[5]}$ \\ [1] Universidade Federal Rural do Semiárido; brunomatheusmsilva@ gmail.com \\ [2] Universidade Federal Rural do Semiárido; lucasvinicius.ctec@ gmail.com \\ ${ }^{[3]}$ Universidade Federal Rural do Semiárido; stephano.mns@ gmail.com \\ [4] Universidade Federal Rural do Semiárido; fkv@ufersa.edu.br \\ [5] Universidade Federal Rural do Semiárido; marceloguerra@ufersa.edu.br
}

Recebido: 10/06/2020;

Aceito: 30/07/2020;

Publicado: 11/09/2020.

Resumo: A matriz energética mundial sofreu várias alterações devido à inserção de novas fontes renováveis de energia nos últimos anos, causada pela preocupação com os impactos socioambientais e políticos provenientes da utilização de combustíveis fósseis. Dentre as fontes sustentáveis de energia pode-se destacar a solar, hídrica e a energia eólica. Visando a importância das fontes renováveis de energia eólica e a necessidade de aprimorar sua eficiência, este artigo apresenta o processo de desenvolvimento de um protótipo de aerogerador de eixo horizontal, com um sistema automatizado que rotaciona o eixo de acordo com a direção do vento. Durante o processo foi dada a devida prioridade à utilização de componentes recicláveis e de baixo custo para a construção do protótipo, onde nos resultados pôde-se observar uma geração de tensão próxima à tensão nominal do motor responsável pela conversão da energia cinética em energia elétrica. O protótipo ainda pode ser utilizado para fins didáticos e como base para futuros estudos e aperfeiçoamento do mesmo.

Palavras-chave: aerogerador, direcionador de vento, eixo horizontal, energia eólica.

Abstract: The world energy matrix has undergone several changes due to the insertion of new renewable energy sources in recent years, caused by the concern about the socio-environmental and political impacts from the use of fossil fuels. Among the sustainable sources of energy, solar, hydro and especially wind power can be highlighted. Aiming at the importance of renewable sources of wind energy and the need to improve their efficiency, this article presents the process of developing a prototype of a horizontal axis wind turbine, with an automated system that rotates the axis according to the wind direction. During the process, due priority was given to the use of recyclable and low-cost components for the construction of the prototype, where the results showed a voltage generation close to the nominal voltage of the engine responsible for the conversion of kinetic energy into electricity. The prototype can still be used for didactic purposes and as a basis for future studies and improvement.

Key-words: wind turbine, wind driver, horizontal axis, wind energy.

\section{INTRODUÇÃO}

A $\mathrm{S}$ fontes renováveis de energia vêm ganhando destaque por serem alternativas às antigas fontes não renováveis, que são esgotáveis e geram diversos impactos negativos no meio ambiente. Nos últimos anos, os debates acerca das mudanças climáticas aumentaram consideravelmente a nível mundial, tendo como pauta principal a redução da emissão de gases provenientes da queima de combustíveis fósseis. Além dos 
problemas ambientais, existem os problemas políticos ligados às fontes não renováveis, já que importantes pontos de extração estão localizados em regiões com grande concentração de conflitos bélicos. Dessa forma, o estudo e desenvolvimento de formas alternativas que possam diversificar a matriz energética mundial cresceram de forma significativa nos últimos anos [1].

O aproveitamento da energia cinética dos ventos não é algo recente. A humanidade aproveita os benefícios dos ventos há mais de 3000 anos, como em atividades agrícolas e marítimas. As duas principais aplicações que são encontradas até os dias de hoje, a saber, os moinhos de vento para bombeamento de água e os aerogeradores para geração de energia elétrica, sendo este último o mais recente e de extrema importância para a matriz de energia elétrica de qualquer país [2]. No Brasil, o incentivo às fontes renováveis de energia elétrica foi impulsionado pelo Programa de Incentivo às Fontes Alternativas (PROINFA). Criado pela lei no 10.438/2002 [3], o PROINFA comtempla as pequenas centrais hidrelétricas, a energia eólica e energia de biomassa, e tem como objetivo aumentar a participação dessas fontes renováveis na matriz elétrica brasileira. Tendo a energia eólica uma significativa participação de empreendimentos no âmbito do PROINFA, em 2020, segundo [5], a matriz elétrica brasileira é formada por $82 \%$ de fontes renováveis, sendo que a eólica já é responsável por aproximadamente $9 \%$ de participação na matriz de energia elétrica, que consiste em algo em torno de $15.500 \mathrm{MW}$ de potência instalada.

Aproveitando os constantes avanços tecnológicos em todo o mundo, é importante o incentivo e o desenvolvimento de pesquisas que visem o aperfeiçoamento dos sistemas responsáveis pelo usufruto de fontes renováveis para geração de energia elétrica. Os aerogeradores de eixo fixo, por exemplo, são instalados em diferentes posições em um parque eólico com o objetivo de aproveitar as variações de direção dos ventos. Um sistema automatizado que possibilite a rotação do eixo dos aerogeradores de acordo com a direção do vento e, portanto, se adapte às suas variações, á algo que vem sendo utilizado nos aerogeradores modernos, pois possibilita maior aproveitamento da energia cinética.

Por todo o exposto, o presente artigo tem como objetivo desenvolver um protótipo de aerogerador de um eixo horizontal com sistema automatizado de direção de vento. Tem-se o intuito de proporcionar o alinhamento prático das informações disponibilizadas por livros didáticos e artigos em geral, assim como o de utilizar o protótipo para aplicações didáticas, pesquisa e aperfeiçoamento.

\section{SISTEMAS ELÉTRICOS}

Os sistemas eólicos são aqueles que utilizam a energia eólica, ou seja, a energia cinética contida nas massas de ar. A partir de equipamentos que convertem a translação das massas de ar em rotação de seus eixos, os sistemas eólicos podem ser utilizados de diversas formas, como no bombeamento de água e na geração de energia elétrica. Os aerogeradores, ou turbinas eólicas, são geradores elétricos que convertem a energia cinética dos ventos em energia elétrica. Apresentando-se como uma alternativa renovável para a geração de energia elétrica, as turbinas eólicas podem ser encontradas de duas formas distintas quanto à posição de seus eixos, sendo estas: turbina de eixo vertical e turbina de eixo horizontal. O modelo adotado para a construção do protótipo foi o de eixo horizontal, eles são os mais simples de serem construídos, apresentam maior estabilidade e rendimento e são os mais utilizados atualmente [5].

\subsection{Aerogerador de eixo horizontal}

Os aerogeradores de eixo horizontal são os mais conhecidos, já que são os mais utilizados mundialmente no ramo da geração de energia elétrica. Eles são projetados de forma que as forças aerodinâmicas do ar movimentem as suas pás, rotacionando o seu rotor, e, dessa forma, convertendo a energia cinética dos ventos 
em mecânica. Os aerogeradores convencionais são formados por 3 pás e giram predominantemente sob forças de sustentação, que atuam em direção perpendicular ao escoamento do vento. Esse modelo se apresenta de forma mais eficiente, pois é capaz de liberar mais potência quando comparado a aerogeradores que funcionam com ação predominante de forças de arrasto, que atuam na mesma direção do escoamento. A Figura 1 apresenta os principais componentes de um aerogerador de eixo vertical [6].

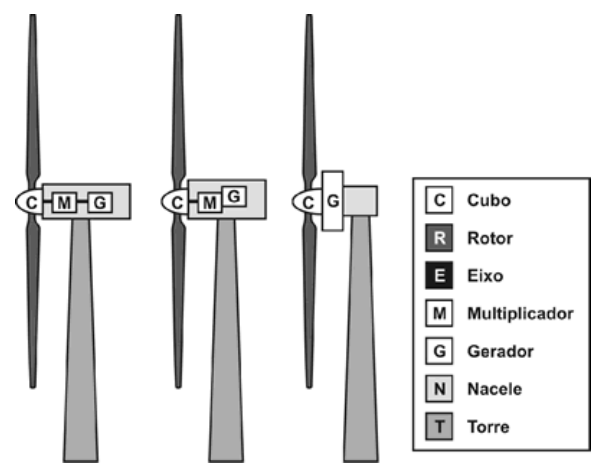

FIGURA 1. Principais componentes de um aerogerador de eixo horizontal.

Os aerogeradores podem ser diferenciados pelo tamanho e pela carcaça que está sobre a torre, chamada de nacele, que pode ou não apresentar caixa multiplicadora, que tem a função de aumentar a frequência angular de rotação do eixo que chega até o gerador. As pás, que estão conectadas ao cubo, são projetadas de forma a captar o vento e converter a translação das massas de ar em rotação, rotacionando o seu eixo que está fixado ao gerador ou à caixa multiplicadora. $\mathrm{O}$ volume das massas de ar que chegam até as pás irá influenciar diretamente na velocidade de rotação do rotor, sendo assim, um aerogerador automatizado de forma a rotacionar o seu eixo de acordo com a direção do vento será mais eficiente do que o de eixo fixo.

\subsection{Sistemas Eólicos no Brasil}

A matriz elétrica brasileira é majoritariamente composta por fontes renováveis, conforme divulgado pelo BIG ANEEL 2020 [5]. Elas são responsáveis por 141.317 MW do total de 172.338 MW de potência instalada, ou seja, $82 \%$ da matriz elétrica brasileira é composta por fontes renováveis de energia. A participação dos sistemas eólicos de geração de energia elétrica no cenário brasileiro consiste em 15.519 MW de potência instalada, e como informado anteriormente corresponde a quase $9 \%$ do total. [5] também fornece os dados dos empreendimentos que ainda estão em fase de construção e dos que a construção ainda não foi iniciada. Esses dados destacam o potencial de crescimento das fontes eólicas na matriz elétrica brasileira, já que, dos empreendimentos em construção, elas apresentam algo em torno de 34\% da potência outorgada, e, dos empreendimentos com construção não iniciada, representam aproximadamente $31 \%$ da potência outorgada. Os dados detalhados podem ser observados na Tabela 1 .

TABELA 1. Fontes utilizadas no Brasil - Construção iniciada e não iniciada [5].

\begin{tabular}{|c|c|c|c|c|}
\hline \hline \multicolumn{5}{|c|}{ Fontes Utilizadas no Brasil } \\
\hline \hline \multirow{2}{*}{ Origem } & \multicolumn{2}{|c|}{ Em construção } & $\%$ & \multicolumn{2}{c|}{ Construção não iniciada } \\
\cline { 2 - 5 } & Potência outorgada & 31,71 & 2.659 .302 & $\%$ \\
\hline Fóssil & 2.458 .405 & 5,3 & 1.328 .175 & 6,26 \\
\hline Biomassa & 411.189 & 17,41 & - & - \\
\hline Nuclear & 1.350 .000 & 6,38 & 1.711 .622 & 8,07 \\
\hline Hídrica & 494.541 & 33,66 & 6.504 .455 & 30,68 \\
\hline Eólica & 2.609 .985 & 5,54 & 8.998 .841 & 42,44 \\
\hline Solar & 429.688 & & & \\
\hline
\end{tabular}




\section{MATERIAIS E MÉTODOS}

O estudo e elaboração do artigo foi dividida em duas etapas visto que por se tratar de uma aplicação prática foram necessários testes prévios para verificar a possibilidade de implementação do protótipo. A primeira etapa foi o desenvolvimento do sketch Arduino, ou seja, as linhas de código de programação que possibilitariam a automação do sistema. Já a segunda etapa foi propriamente a construção e montagem física do protótipo proposto.

\subsection{Desenvolvimento do código de programação e testes virtuais}

A programação foi, primeiramente, desenvolvida utilizando um site criado pela AutoDesk®, o TinkerCad. O TinkerCad é uma plataforma online e gratuita que disponibiliza ferramentas de softwares para a elaboração de projetos como Design 3D, eletrônica e programação livre, para utilizar esta ferramenta basta acessar o link da plataforma em [8] e preencher sua inscrição.

Com o auxílio da plataforma foi possível escrever o código de programação de forma rápida e simples além de testar a aplicação de forma virtual, antecipando os testes físicos e reais, dessa forma prevenindo riscos e falhas no sistema. O código primário desenvolvido na plataforma do TinkerCad é feito em blocos (Figura 2) permitindo maior agilidade ao processo.

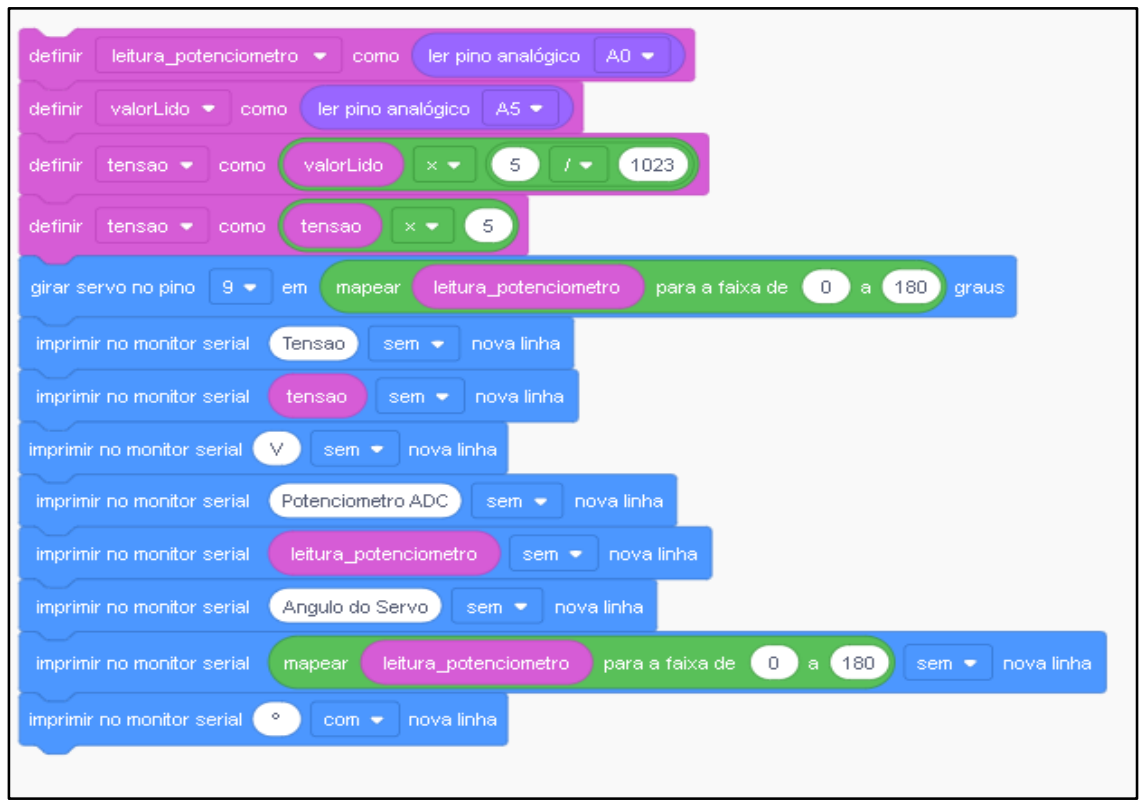

FIGURA 2. Bloco de programação desenvolvido no TinkerCad (Autoria própria).

Após a validação do TinkerCad foi possível implementar a rotina para Arduino, possibilitando assim a comunicação entre as duas plataformas de desenvolvimento. Além do código em blocos, a plataforma também permite que sejam implementados dispositivos eletrônicos virtuais para que se possa simular e extrair resultados de forma online. Nos testes realizados foram utilizadas a placa Arduino UNO (1), um potenciômetro de $20 \mathrm{~K} \Omega$ (Quilo-Ohm) (2), um micro servo motor (3) e um motor de corrente contínua (4), como ilustrado na Figura 3. 


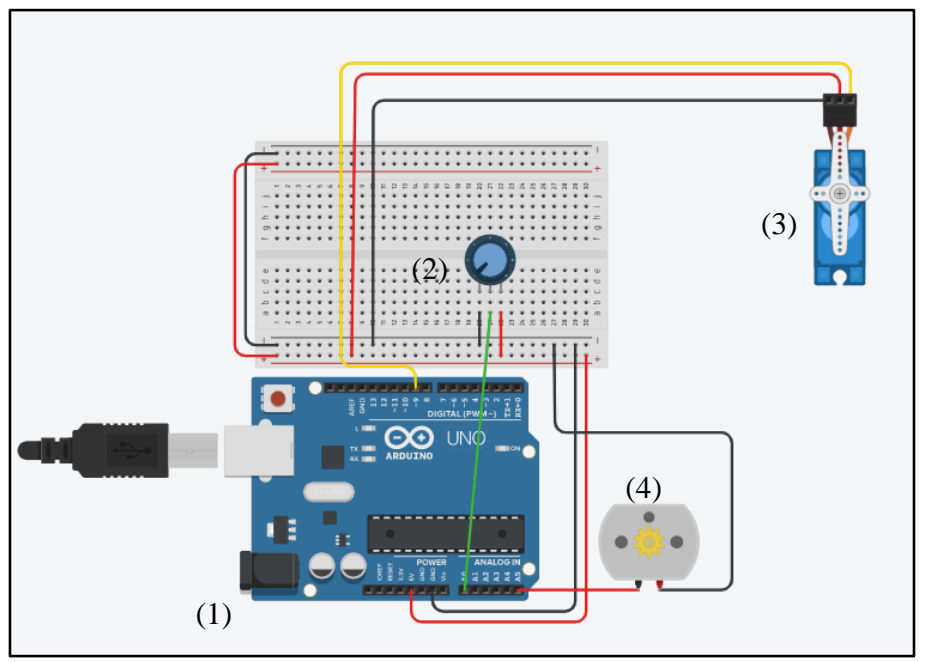

FIGURA 3. Simulação do circuito eletrônico de forma virtual: (1) Arduíno uno; (2) Potenciômetro; (3) Micro servo motor; (4) Motor DC (Autoria própria).

Com o código exposto e os dispositivos alocados de forma correta é possível entender o funcionamento do sistema e a lógica de programação implementada seguindo o sketch da Figura 4.

\begin{tabular}{|c|c|}
\hline 1. \#include <Servo.h> & 17. servo_9.write(map(leitura_potenciometro, 0 , \\
\hline 2. \#include $\langle$ Wire.h $>$ & $1023,0,180))$ \\
\hline 3. int valorLido $=0$ & 18. valorLido $=$ analogRead $(\mathrm{A} 5)$; \\
\hline 4. float tensao $=0$ & 19. tensao $=$ valorLido $*(5.0 / 1023.0)$ \\
\hline 5. int leitura_potenciometro $=0$; & 20. tensao $=$ tensao $* 5$ \\
\hline 6. Servo servo_9; & 21. Serial.print("Tensao: "); \\
\hline 7. void setup() & 22. Serial.print(tensao); \\
\hline 8. \{ & 23. Serial.print(" V"); \\
\hline 9. $\operatorname{pinMode}(\mathrm{A} 0$, INPUT); & 24. Serial.print(" Potenciometro ADC: "); \\
\hline 10. servo_9.attach(9); & 25. Serial.print(leitura_potenciometro); \\
\hline 12. Serial.begin(19200); & 26. Serial.print(" Angulo do Servo: "); \\
\hline 13. $\}$ & 27. Serial.print(map(leitura_potenciometro,0, 1023, \\
\hline 14. void loop() & $0,180)$ ); \\
\hline 15. \{ & 28. Serial.println("०"); \\
\hline \multirow[t]{2}{*}{ 16. leitura_potenciometro $=$ analogRead(A0); } & 29.delay(100); \\
\hline & \} \\
\hline
\end{tabular}

FIGURA 4. Sketch de implementação do sistema para a plataforma Arduíno (Autoria Própria).

De forma geral é possível entender o funcionamento do sistema como, o potenciômetro sendo um sensor direcionador de vento que, conectado a placa Arduino, irá fornecer variação na sua resistência e consequentemente variação de tensão nas portas da placa. Essa variação será interpretada e traduzida pelo Arduino e transmitida ao servo motor proporcionalmente a conversão de escala de bit 0 a 1023 para escala de ângulo de giro de 0 a $180^{\circ}$, permitindo que o eixo do aerogerador acoplado ao servo motor se alinhe com a massa de ar automatizando o sistema de rotação. Além disso, o motor DC que funcionará como gerador de energia eólica está conectado à porta analógica Arduino que realizará a leitura dos seus níveis de tensão, permitindo identificar o aumento ou redução em função da direção em que o eixo acoplado se encontra em frente a massa de ar. 


\subsection{Construção física do protótipo}

O protótipo de aerogerador de eixo horizontal foi confeccionado utilizando materiais de baixo custo, pois o propósito maior do desenvolvimento deste trabalho está centrado na análise de controle da rotação do eixo da nacele do aerogerador em direção a maior massa de ar disponível. Como já mencionado anteriormente, para a construção do protótipo foram utilizados materiais de baixo custo, no caso, uma rolha de garrafa de vinho, uma peça de madeira circular de $21 \mathrm{~cm}$ de comprimento e $2 \mathrm{~cm}$ de diâmetro, uma base quadrada em MDF com dimensão $10 \mathrm{~cm}$ x $8,5 \mathrm{~cm}$, um motor de corrente contínua utilizado em controles de vibração com tensão nominal de 5,5 V, um micro servo motor 9g SG90, um jumper (Arduino) macho de $6 \mathrm{~cm}$ e $10 \mathrm{~cm}$, folha de papel A4, fita adesiva e uma garrafa pet reaproveitada de $\mathrm{X} \mathrm{ml}$.

As hélices foram confeccionadas utilizando o material extraído da garrafa pet, e foram cortadas igualmente em 3 retângulos com 1,5 cm de altura e $4 \mathrm{~cm}$ de comprimento. Após essa etapa, as mesmas foram acopladas a uma rolha de garrafa de vinho utilizando-se cola, esta tinha função de trabalhar como o eixo de acoplamento das hélices (pás do gerador) no rotor do motor DC. O motor DC, que funciona como o próprio gerador eólico, foi acoplado com cola e fita adesiva ao micro servo motor para que a rotação fosse realizada quando necessária, com o intuito de orientação em relação a direção do vento. Em seguida, o micro servo motor foi acoplado ao topo da madeira (revestida por papel A4) que tinha a função de ser a torre do aerogerador. O mesmo foi fixado com cola e fita adesiva também. Os componentes que constituem o protótipo estão dispostos e identificados na Figura 5.

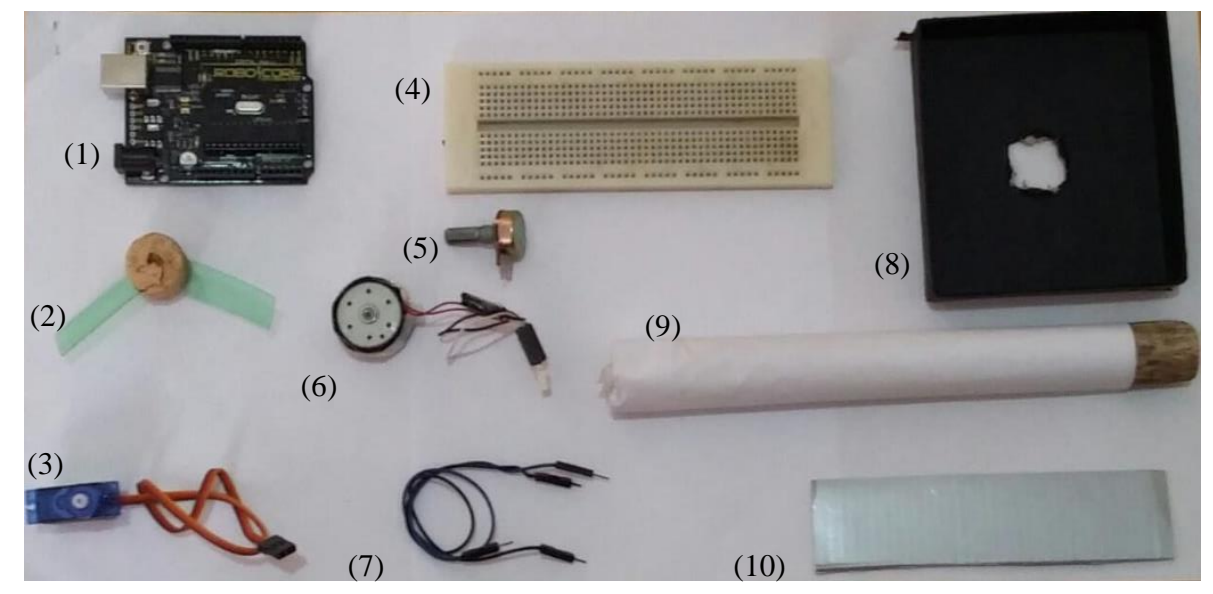

FIGURA 5. Componentes para montagem do protótipo de aerogerador: (1) Arduino Uno. (2) Hélices confeccionadas de garrafa pet. (3) Micro servo motor 9g SG90. (4) Protoboard. (5) Potenciômetro de $20 \mathrm{~K} \Omega$. (6) Motor DC 5,5 V. (7) Jumper conector. (8) Base do aerogerador em MDF. (9) Torre do aerogerador confeccionada de madeira circular. (10) Recorte de papelão utilizado para direcionar a massa de ar (Autoria própria).

As conexões elétricas tanto do motor DC quanto do micro servo motor foram conectadas ao Arduino para que houvesse a leitura e comunicação entre os dispositivos. Para a construção do direcionador de vento foi utilizado um recorte em papelão com $12 \mathrm{~cm}$ de comprimento e $4 \mathrm{~cm}$ de altura, que foi acoplado ao pino do potenciômetro para que, em contato com a massa de ar, o papelão transferisse o esforço ao potenciômetro e conseguisse rotacionar seu pino fazendo-o variar em função do vento, o resultado final do protótipo desenvolvido pode ser observado na Figura 6. 


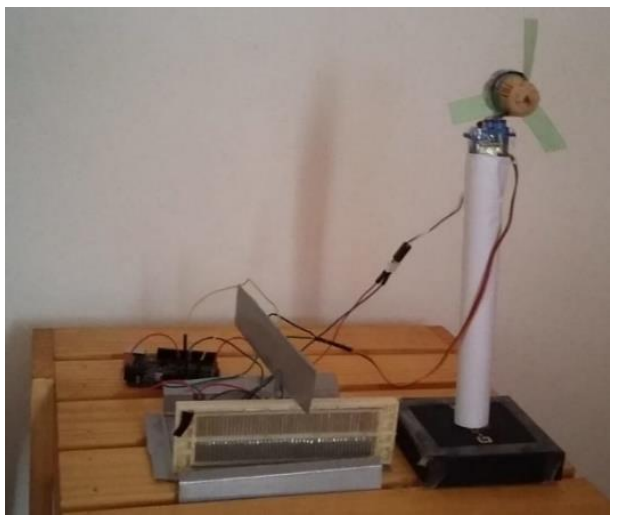

(a)

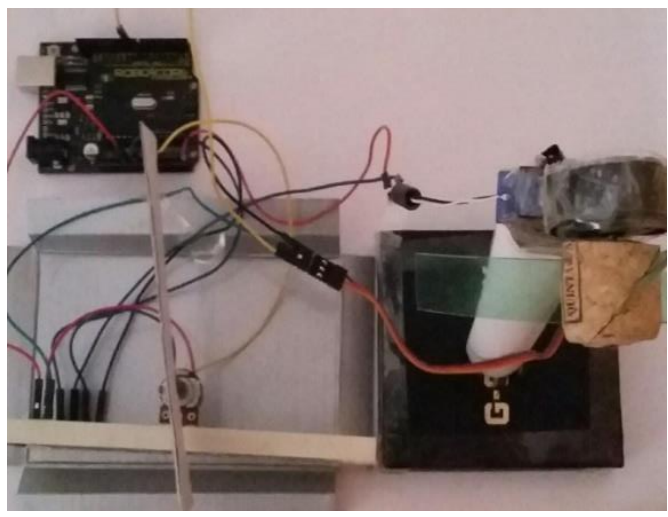

(b)

FIGURA 6. Protótipo de aerogerador montado e conectado: (a) Vista frontal do protótipo de aerogerador.

(b) Vista superior do protótipo de aerogerador (Autoria própria).

\section{RESULTADOS}

Ao final da construção física do protótipo, foi possível observar o funcionamento do sistema e analisar as variáveis do projeto. Os testes foram feitos utilizando um ventilador para simular as massas de ar que interagem com o sistema. Ao ligar o ventilador, a massa de ar concentrada entra em contato com o sensor de direção de vento, representado pelo conjunto do papelão e potenciômetro. À medida que o sensor tende a se alinhar com a massa de ar, rotacionando em seu próprio eixo, o servo motor se alinha ao eixo do potenciômetro e massa, como era esperado. Pelo monitor serial foi possível observar que, à medida que o aerogerador rotaciona em direção a massa de ar, os níveis de tensão em sua saída aumentam e variam devido à interferência do sistema, como mostra a Figura 7.

\begin{tabular}{|c|c|c|c|c|c|c|c|}
\hline \multicolumn{8}{|l|}{2 coms } \\
\hline Tensao: & $0.00 \mathrm{~V}$ & Potenciometro & ADC: & 367 & Angulo o & do Servo: & $64^{*}$ \\
\hline Tensao: & $0.00 \mathrm{~V}$ & Potenciometro & ADC: & 367 & Angulo o & do Servo: & $64^{\circ}$ \\
\hline Tensao: & $0.00 \mathrm{~V}$ & Potenciometro & ADC: & 367 & Angulo & do Servo: & $64^{*}$ \\
\hline Tensao: & $0.00 \mathrm{~V}$ & Potenciometro & $A D C:$ & 367 & Angulo & do Servo: & $64^{\circ}$ \\
\hline Tensao: & $0.00 \mathrm{v}$ & Potencionetro & ADC: & 367 & Angulo & do servo: & $64^{\circ}$ \\
\hline Tensao: & $0.00 \mathrm{~V}$ & Potenciometro & $A D C:$ & 367 & Angulo & do Servo: & $64^{\circ}$ \\
\hline Tensao: & $0.00 \mathrm{~V}$ & Potenciometro & $A D C:$ & 367 & Angulo & do Servo: & $64^{\circ}$ \\
\hline Tensao: & $0.00 \mathrm{v}$ & Potenciometro & $A D C:$ & 367 & Angulo & do Servo: & $64^{\circ}$ \\
\hline Tensao: & $0.00 \mathrm{~V}$ & Potenciometro & $A D C:$ & 367 & Angulo o & do Servo: & $64^{\circ}$ \\
\hline Tensao: & $0.00 \mathrm{~V}$ & Potencionetro & $A D C:$ & 367 & Angulo & do Servo: & $64^{\circ}$ \\
\hline Tensao: & $0.00 \mathrm{~V}$ & Potencionetro & $A D C:$ & 367 & Angulo & do Servo: & $64^{\circ}$ \\
\hline Tensao: & $0.00 \mathrm{v}$ & Potenciometro & $A D C:$ & 367 & Angulo & do Servo: & $64^{\circ}$ \\
\hline & $0.00 \mathrm{~V}$ & neionetro & ADC: & 367 & Angulo & do Servo: & $64^{\circ}$ \\
\hline Tensao: & $0.00 \mathrm{v}$ & Potenciometro & $A D C$ : & 367 & Angulo & do Servo: & $64^{\circ}$ \\
\hline
\end{tabular}

\begin{tabular}{|c|c|c|c|c|c|c|c|}
\hline OMS & & & & & & & \\
\hline Tensao: & $1.08 \mathrm{~V}$ & Potencionetro & ADC: & 578 & Angulo do & Servo: & $101^{*}$ \\
\hline Tensao: & $2.27 \mathrm{~V}$ & Potencioxetro & ADC: & 600 & Angrulo do & Servo: & $105^{\circ}$ \\
\hline Tensao: & $5.33 \mathrm{~V}$ & Potencionetro & ADC: & 600 & Angulo do & Servo: & $105^{\circ}$ \\
\hline Tensao: & $4.20 \mathrm{~V}$ & Potencionetro & ADC: & 643 & Angulo do & Servo: & $113^{\circ}$ \\
\hline Tensao: & $4.52 \mathrm{~V}$ & Potencionetro & $A D C:$ & 664 & Angulo do & Servo: & $116^{\circ}$ \\
\hline Tensao: & $4.94 \mathrm{~V}$ & Potencionetro & $\mathrm{ADC}:$ & 684 & Angulo do & Servo: & $120^{\circ}$ \\
\hline Tensao: & $4.67 \mathrm{v}$ & Potencionetro & $A D C:$ & 702 & Angulo do & Servo: & $123^{\circ}$ \\
\hline Tensao: & $4.62 \mathrm{~V}$ & Potencionetro & $\mathrm{ADC}:$ & 720 & Angulo do & Servo: & $126^{\circ}$ \\
\hline Tensao: & $4.33 \mathrm{~V}$ & Potencionetro & $\mathrm{ADC}:$ & 732 & Angulo do & Servo: & $128^{\circ}$ \\
\hline Tensao: & $4.64 \mathrm{~V}$ & Potenciozetro & $\mathrm{ADC}:$ & 742 & Angulo do & Servo: & $130^{\circ}$ \\
\hline Tensao: & $4.20 \mathrm{~V}$ & Potencionetro & $\mathrm{ADC}:$ & 750 & Angulo do & Servo: & $131^{\circ}$ \\
\hline Tensao: & $4.47 \mathrm{~V}$ & Potencionetro & ADC: & 758 & Angulo do & Servo: & $133^{\circ}$ \\
\hline Tensao: & $5.23 \mathrm{~V}$ & Potencionetro & ADC: & 767 & Angulo do & Servo: & $134^{\circ}$ \\
\hline Tensao: & $5.18 \mathrm{~V}$ & Potencionetro & $A D C:$ & 744 & Angulo do & Servo: & $130^{\circ}$ \\
\hline Tensao: & $4.37 \mathrm{~V}$ & Potencioxetro & ADC: & 776 & Angulo do & Servo: & $136^{\circ}$ \\
\hline Tensao: & $3.91 \mathrm{~V}$ & Potencionetro & ADC: & 786 & Angulo do & Servo: & $138^{\circ}$ \\
\hline ensao: & $3.96 \mathrm{v}$ & Potencioxetro & ADC: & 788 & Angulo do & Servo: & $138^{\circ}$ \\
\hline Tensao: & $3.62 \mathrm{~V}$ & Potencionetro & $\mathrm{ADC}:$ & 790 & Angulo do & Servo: & $139^{\circ}$ \\
\hline Tensao: & $3.47 \mathrm{~V}$ & Potencionetro & ADC: & 789 & Angulo do & Servo: & $138^{\circ}$ \\
\hline Tensao: & $3.20 \mathrm{~V}$ & Potencionetro & $A D C:$ & 789 & Angulo do & Servo: & $138^{\circ}$ \\
\hline & $3.54 \mathrm{~V}$ & Potenciouetro & ADC: & 789 & Angulo do & Servo: & $138^{\circ}$ \\
\hline Tensao: & $3.35 \mathrm{~V}$ & Potencionetro & ADC: & 789 & Angulo do & Servo: & $138^{\circ}$ \\
\hline Tensao: & $3.32 \mathrm{~V}$ & Potencionetro & ADC: & 789 & Angulo do & Servo: & $138^{\circ}$ \\
\hline Tensao: & $3.30 \mathrm{~V}$ & Potencionetro & $\mathrm{ADC}:$ & 790 & Angulo do & Servo: & $139^{\circ}$ \\
\hline Tensao: & $3.03 \mathrm{~V}$ & Potencionetro & ADC: & 790 & Angulo do & Servo: & $139^{\circ}$ \\
\hline Tensao: & $3.32 \mathrm{~V}$ & Potencionetro & ADC: & 789 & Angulo do & Servo: & $138^{\circ}$ \\
\hline
\end{tabular}

(a)

(b)

FiguRA 7. Níveis de Tensão, posição e angulação registrados pelo monitor serial: (a) Níveis antes do contato com massas de ar. (b) Níveis após o sistema entrar em contato com massas de ar (Autoria própria).

Foi identificado que, tanto no início quanto no final do direcionamento em relação a massa de ar, que existe uma defasagem de rotação do eixo do potenciômetro em relação a rotação do micro servo motor, ou seja, no momento em que a massa de ar entra em contato com o potenciômetro, e o rotaciona, era esperado que 
imediatamente o micro servo motor também rotacionasse, se ajustando quase que instantaneamente a sua posição, no entanto isso não ocorreu.

A partir dessa observação e a realização de alguns testes, constatou-se que esse problema poderia ser resolvido com a redução do valor de resistência do potenciômetro de $20 \mathrm{~K} \Omega$ para $5 \mathrm{~K} \Omega$, consequentemente diminuindo o seu grau de liberdade de rotação e permitindo que com uma quantidade menor de tensão o micro servo motor conseguisse rotacionar.

Para testar a tensão gerada pelo protótipo, foi utilizado um equipamento secador de cabelo com duas intensidades distintas, onde as velocidades foram medidas com um anemômetro. A velocidade mais baixa foi de $7 \mathrm{~m} / \mathrm{s}$, gerando uma tensão de saída no gerador de aproximadamente 2,3V. Já a velocidade mais alta, que foi $10,5 \mathrm{~m} / \mathrm{s}$, proporcionou aproximadamente $4,8 \mathrm{~V}$ como tensão de saída, sendo $5,5 \mathrm{~V}$ a tensão nominal do gerador utilizado.

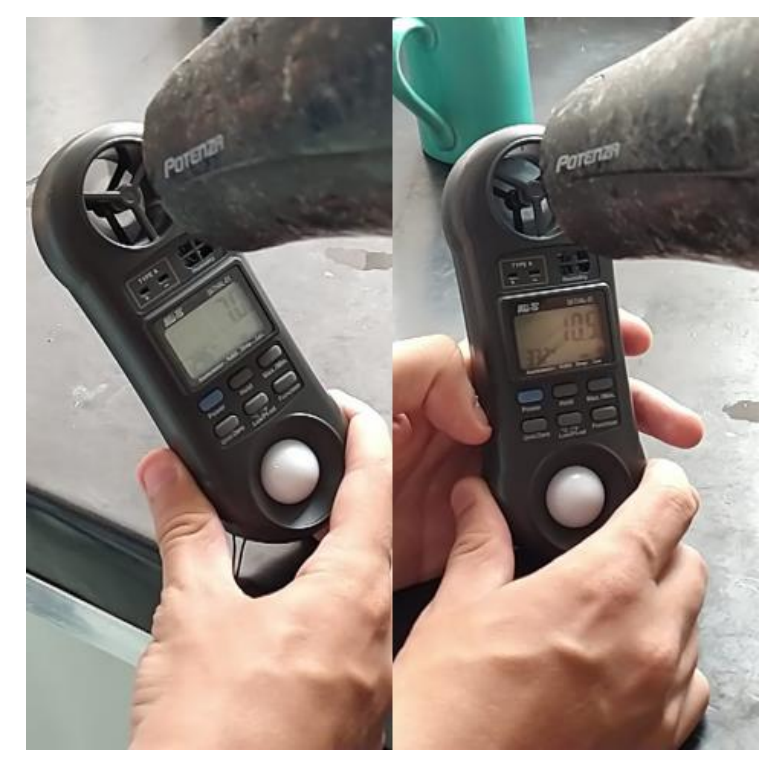

FIGURA 8. Testes da velocidade do vento com o anemômetro (Autoria própria).

\section{CONCLUSÃO}

Mediante a finalização do protótipo, os resultados foram bem próximos do esperado. Como o processo envolveu a construção do aerogerador e do sensor de direção de vento, a análise dos resultados foi realizada a partir da observação dos dois mecanismos em funcionamento. Com relação à tensão de saída do gerador, foi possível atingir 4,8V com velocidade de $11 \mathrm{~m} / \mathrm{s}$, sendo esse valor bem perto da tensão nominal de funcionamento, que é de $5,5 \mathrm{~V}$, o que pode ser definido como um ponto bastante positivo. Vale ressaltar que os testes foram realizados a partir de uma simulação de vento gerado por um secador de cabelo, ou seja, o estudo do funcionamento ao ar livre também é possível, mas vai depender das condições climáticas do local durante o período de análise. O sensor de direção do vento também se mostrou bastante efetivo durante a observação, porém o tempo de resposta entre a rotação do sensor e do micro servo motor foi um pouco mais lento que o esperado, mas nada que influenciasse negativamente o funcionamento geral do protótipo.

O custo do material utilizado para a construção do protótipo foi, de maneira geral, um investimento de baixo custo e acessível, o Arduino uno no valor de R\$89,90, Micro Servo 9g SG90 R \$17,90, Motor DC R\$ 6,90, Potenciômetro R \$3,90, Protoboard R \$18,90, Jumper R \$14,90 totalizando um custo aproximado de R \$150. Entretanto como exposto anteriormente, muitos dos materiais utilizados foram reaproveitados, e a parte eletrônica foi providenciada pelos membros do grupo o que reduziu o custo do protótipo. 
Com os resultados obtidos, o aerogerador consegue ser um instrumento bastante útil para análise prática do processo de conversão de energia cinética dos ventos em elétrica, podendo ser utilizado para fins didáticos. O mesmo também está disponível para melhorias, como a correção do tempo de resposta entre o direcionador de vento e o micro servo motor e a sua aerodinâmica, sendo possível a adaptação para a obtenção dos mesmos resultados de geração quando utilizado sob massas de ar com menores velocidades. Sendo assim, o protótipo oferece diversos caminhos para seu estudo e aprimoramento.

\section{REFERÊNCIAS}

[1] DE BARROS, E. V. A Matriz Energética Mundial E A Competitividade das Nações: bases de uma nova geopolítica. Engevista 2007, Volume 9, 10 páginas.

[2] MARTINS, F. R.; GUARNIERI, R. A.; PEREIRA, E. B. O Aproveitamento da Energia Eólica. $R B E F$ 2008, Volume 30, 13 páginas.

[3] LEI $\mathrm{N}^{\mathrm{o}}$ 10.438, DE 26 DE ABRIL DE 2002. Disponível online: <http://www.planalto.gov.br/ccivil_03/leis/2002/L10438.htm> (acesso em 07/01/2020).

[4] ELETROBRAS. Geração Líquida Mensal Rateada por Contrato. Disponível online: <https://eletrobras.com/pt/AreasdeAtuacao/programas/proinfa/2019_Dados_de_Geracao_das_CGEE_pa rticipantes_do_PROINFA-geracao_liquida.pdf> (acesso em 10/05/2020).

[5] BIG. Banco de Informações de Geração da ANEEL. Matriz de Energia Elétrica. Disponível em: <https://www2.aneel.gov.br/aplicacoes/capacidadebrasil/Combustivel.cfm> (acesso em 14/05/2020).

[6] CARVAlHO, B. C. Desenvolvimento de Modelo Computacional de Sistemas Eólicos Utilizando Geradores Síncronos para Estudos de Desempenho no Contexto da Qualidade da Energia Elétrica. Tese (Doutorado em Engenharia Elétrica), Universidade Federal de Uberlândia, Uberlândia, 2006.

[7] CENTRO DE REFERÊNCIA PARA AS ENERGIAS SOLAR E EÓLICA SÉRGIO DE S. BRITO CRESESB. Tipos de Aerogeradores para Geração de Energia Elétrica. Disponível online: <http://www.cresesb.cepel.br/index.php?section=com_content\&lang=pt\&cid=231> (acesso em 08/01/2020).

[8] Plataforma Tinkercad. Disponível em: < https://www.tinkercad.com > (acesso em: 20/12/2019).

[9] Arduino. Disponível em: <https://www.arduino.cc/> (acesso em 23/12/2019). 